ARTICLE

\title{
Structural basis of LAIR1 targeting by polymorphic Plasmodium RIFINs
}

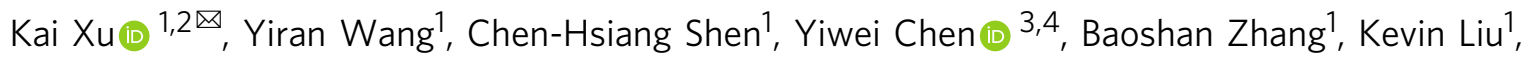
Yaroslav Tsybovsky ${ }^{5}$, Shuishu Wang (10 1, S. Katie Farney ${ }^{1}$, Jason Gorman (1) ${ }^{1}$, Tyler Stephens ${ }^{5}$, Raffaello Verardi (1) 1, Yongping Yang1, Tongqing Zhou (1) 1, Gwo-Yu Chuang1, Antonio Lanzavecchia (1) 3,6, Luca Piccoli (i) ${ }^{3} \&$ Peter D. Kwong (10) ${ }^{1 凶}$

RIFIN, a large family of Plasmodium variant surface antigens, plays a crucial role in malaria pathogenesis by mediating immune suppression through activation of inhibitory receptors such as LAIR1, and antibodies with LAIR1 inserts have been identified that bind infected erythrocytes through RIFIN. However, details of RIFIN-mediated LAIR1 recognition and receptor activation have been unclear. Here, we use negative-stain EM to define the architecture of LAIR1-inserted antibodies and determine crystal structures of RIFIN-variable 2 (V2) domain in complex with a LAIR1 domain. These structures reveal the LAIR1-binding region of RIFIN to be hydrophobic and membrane-distal, to exhibit extensive structural diversity, and to interact with RIFIN-V2 in a one-to-one fashion. Through structural and sequence analysis of various LAIR1 constructs, we identify essential elements of RIFIN-binding on LAIR1. Furthermore, a structure-derived LAIR1-binding sequence signature ascertained >20 LAIR1binding RIFINs, including some from $P$. falciparum field strains and Plasmodium species infecting gorillas and chimpanzees.

\footnotetext{
${ }^{1}$ Vaccine Research Center, National Institute of Allergy and Infectious Diseases, National Institutes of Health, Bethesda, MD, USA. ${ }^{2}$ Department of Veterinary Biosciences, College of Veterinary Medicine, The Ohio State University, Columbus, OH, USA. ${ }^{3}$ Institute for Research in Biomedicine, Università della Svizzera italiana, Bellinzona, Switzerland. ${ }^{4}$ Institute of Microbiology, ETH Zurich, Wolfgang-Pauli-Strasse 10, Zurich, Switzerland. ${ }^{5}$ Electron Microscopy Laboratory, Cancer Research Technology Program, Leidos Biomedical Research, Inc., Frederick National Laboratory for Cancer Research, Frederick, MD, USA.

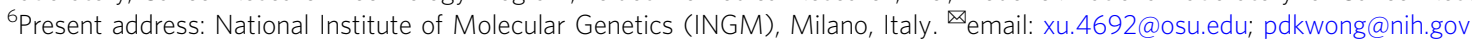


$\mathrm{M}$ alaria infects over 200 million people and kills over 400,000 each year ${ }^{1}$. Its causative agents, Plasmodium parasites, utilize multiple mechanism to evade the human immune response, including the expression of variable surface antigens on Plasmodium-infected erythrocytes (IEs) ${ }^{2}$. With over 150 members, RIFIN comprises one of the larger families of Plasmodium variant surface antigens ${ }^{3,4}$ and sequesters parasites by rosetting group A red blood cells, resulting in the development of severe malaria ${ }^{5}$. RIFIN also mediates immune suppression through activation of inhibitory immune receptors including leukocyte-associated immunoglobulin-like receptor 1 (LAIR1) and leukocyte immunoglobulin-like receptor B1 (LILRB1 $^{6-8}$. Notably, human monoclonal antibodies that contain LAIR1-domain insertions in either switch region or between $\mathrm{V}$ and DJ regions have been isolated from donors, who have had malaria $^{8-10}$. Some of these antibodies, including MGC34 ${ }^{8}$ and MGD2 $1^{8}$, acquire mutations in the LAIR1 domain during antibody maturation to remove self-reactivity for collagen and to increase affinity for $P$. falciparum isolates through interactions with RIFIN $^{8}$.

To define the structural basis underpinning interactions between wild-type and antibody-inserted LAIR1 and polymorphic RIFIN, we investigated the overall architecture of three types of LAIR1-inserted antibodies, determined crystal structures of LAIR1 in complex with the RIFIN-interactive regions from two RIFINs (PF3D7_1040300 and PF3D7_0401300), and used the resultant structural information to derive a LAIR1-binding signature and to identify RIFINS from diverse Plasmodium species capable of recognizing LAIR1.

\section{Results}

Structural definition of RIFIN-LAIR1 interaction. LAIR1containing antibodies have distinct insertion modes, including type- 1 insertion between $\mathrm{V}$ and $\mathrm{DJ}$ junctions in CDR $\mathrm{H} 3$ region, type-2 insertion in the $\mathrm{S}$ region between $\mathrm{VH}$ and $\mathrm{CH} 1$ region, and type-3 insertion in the $\mathrm{S}$ region with deletion of $\mathrm{VH}$ and $\mathrm{CH} 1$ gene 9 . To gain insight into these three insertion architectures, we determined structures for all three types (Supplementary Fig. 1a-d and Supplementary Table 1). Despite differences in architecture and flexibility, RIFIN binding capacity of LAIR1insertion appeared similar as assessed by co-IP and electron microscopy (EM) analyses of a chimeric MGJ5/MGD21 antibody in complex with RIFIN (Supplementary Fig. 1e).

To facilitate structural determination of the RIFIN-V2-LAIR1 interaction, we took advantage of the affinity maturation of the LAIR1 ectodomain from antibody MGD21, which increases its affinity to RIFIN (PF3D7_1040300) to $6.2 \mathrm{nM}$, from lower than 1 $\mu \mathrm{M}$ (Supplementary Fig. 2). Due to its hydrophobicity, RIFIN V2 expresses poorly and has low solubility. We therefore designed a strategy of co-expression and co-purification to obtain the complexes of LAIR1-positive ${ }^{8}$ RIFIN-V2 (PF3D7_1040300, RIF1; and PF3D7_1400600, RIF2) with either LAIR1 ectodomain or MGD21 Fab (Supplementary Fig. 3a, b). The crystal structure of the RIFIN V2 (PF3D7_1040300) in complex with LAIR1 extended to $2.7-\AA$ resolution (Supplementary Table 1 ), while negative-stain (ns)-EM 3D reconstruction of the MGD21 Fab complex with RIFIN V2 (PF3D7_1400600) indicated the Fab region not to be involved in RIFIN binding (Supplementary Fig. 4).

The RIFIN-V2-LAIR1 complex resembled a razor with an exchangeable cartridge, where RIFIN V2 is the "handle" and LAIR1 is the "cartridge" (Fig. 1a). The RIFIN-V2 portion of the complex comprised 6 helices (a1-a6) (Supplementary Fig. 5a). Two longer helices, $\alpha 1$ and $\alpha 6$, and two shorter helices, $\alpha 3$ and $\alpha 4$, formed a four-helix bundle with helices $\alpha 2$ and $\alpha 5$ packed perpendicularly to the bundle. Helix $\alpha 5$ and its adjacent loops ( $\alpha 4-\alpha 5$ and $a 5-\alpha 6)$ were connected by a disulfide bond (C254$\mathrm{C} 265$ ) between $\alpha 4$ and $\alpha 5$; this region (hereafter referred to as the apex region) is likely located distally from the membrane, as the carboxyl terminus of the $\mathrm{V} 2$ region connects directly to the transmembrane. Comparison to a previously determined structure of RIFIN-V2 (PDB $6 Z^{2} X^{7}$ ) revealed only $\sim 10 \%$ sequence identity and extensive variation in length of helices (Supplementary Fig. $5 c, d)$. The LAIR1 ectodomain portion of the complex comprised two $\beta$ sheets along the lines of a standard V-type immunoglobulin fold ${ }^{11}$, with a disulfide bond (C133-C185) connecting strands $\mathrm{B}$ and $\mathrm{F}$ and linking the two $\beta$ sheets (Fig. 1a). Comparison to the previously determined structure of ligand-free LAIR1 (PDB 3KGR ${ }^{11}$ ) suggested the binding of RIFIN to induce conformational alterations in the C-C' loop (Fig. 2a), although their overall structures were essentially identical with a rootmean-square deviation (rmsd) of $0.41 \AA$ over all $\mathrm{Ca}$ atoms. Interestingly, the interface between RIFIN-V2 and LAIR1 buried a surface area of $917 \AA^{2}$ on each protein. The interface consisted of backbone-mediated hydrogen bonds and extensive hydrophobic interactions with highly complementary shapes matching between RIFIN and LAIR1. The RIFIN-V2 apex clamps the side of LAIR1, interacting with loop C-C', strand C, and the loop neighboring strand $\mathrm{F}$. Residues on RIFIN involved in binding LAIR1 included C254, S255, A256, T257 on loop a4-a5, C265, V266, R268 on a5, and P275, M281 on loop a5-a6 (Fig. 1b, c). Interface residues on the LAIR1 included T141, R143 on strand C, R146, Y150, L151 on loop C-C', Y152, S153 on strand C', Y188, W193 in the loop next to strand F. Hydrophobic stacking was observed between LAIR1 Y150, L151 and RIFIN I261, C254C265 disulfide bond. Planar side chain stacking was observed between Y188, W193, R143, Y152 of LAIR1, and R268 and C254C265 disulfide bond of RIFIN (Fig. 1c, and Supplementary Fig. 5f).

The revealed mode of RIFIN interaction with a lateral side of the LAIR1-immunoglobulin domain is strikingly different from the recently determined structure of RIFIN-V2 (PF3D7_1254800) in complex with the inhibitory receptor, LILRB1 $^{7}$ (Supplementary Fig. 5c-d). Collectively, these results provide atomic-level details of RIFIN interaction with LAIR1 and exemplify both the remarkable diversity of RIFINs as well as the distinct modes of RIFIN interaction with cellular factors.

Structure-function analysis of LAIR1-RIFIN interaction. To gain insight into the role of LAIR1 affinity maturation in LAIR1RIFIN interaction, we designed a series of LAIR1 constructs with accumulating mutations in the RIFIN-binding interface, starting from unmutated (native) LAIR1 and extending to the affinitymatured LAIR1 in MGD21, and assessed their binding to RIFIN by co-immunoprecipitation (Fig. 2b, c). Native LAIR1 has an $\mathrm{N}$ linked glycosylation sequon at residue N153, which can be deglycosylated by Endoglycosidase $\mathrm{H}$ (EndoH) treatment (compare the position of LAIR1 in lanes 2 and 3 with that in other lanes in Fig. 2c upper and lower left panel). We found an N153S deglycosylating mutation at the binding interface to enhance RIFIN binding. Mutation T151L hydrophobically interacted with C254C265 in RIFIN V2 (Fig. 1c). Both N153S and T151L LAIR1 mutations substantially increased affinity to RIFIN. By contrast, affinity maturation changes at positions 161,190 , and 191, which are only involved in collagen binding ${ }^{11}$, had no detectable impact on RIFIN binding (Fig. 2c).

The binding footprints of collagen and RIFIN on LAIR1 were distinct but overlapping (Fig. 2d). Several putative critical LAIR1 residues involved in collagen binding, such as P190 and P191, were mutated in LAIR1-inserted antibodies including MGD21, 


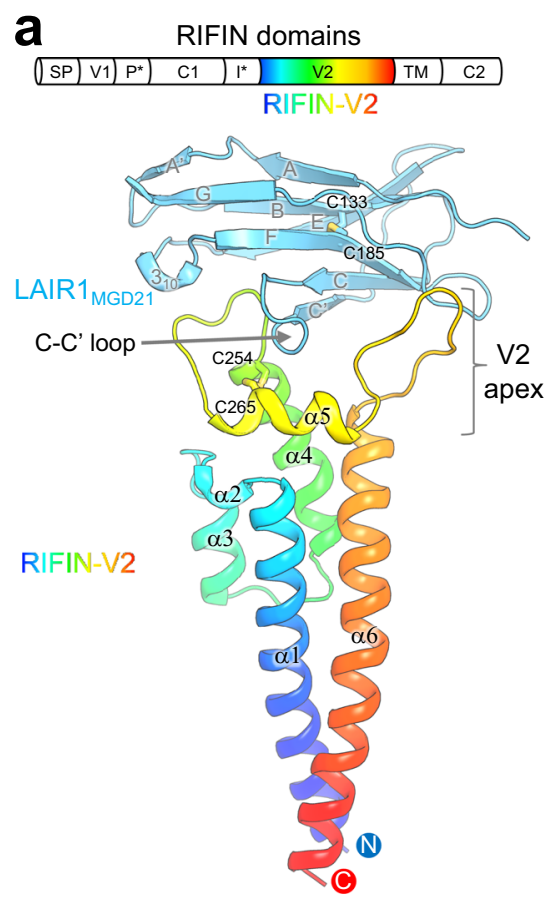

b
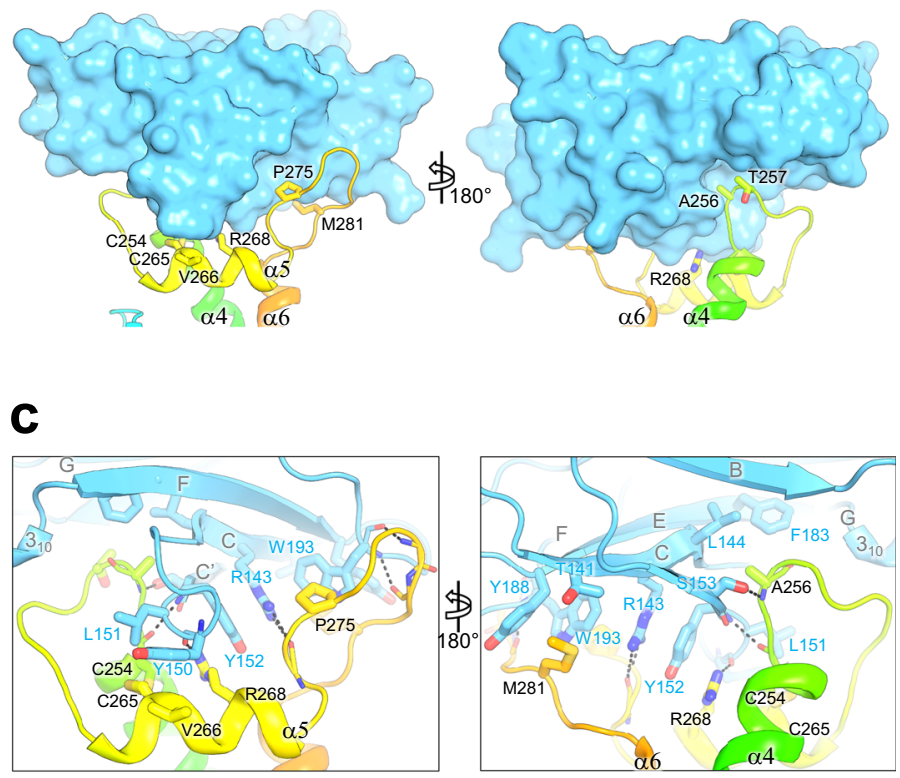

Fig. 1 Structure of RIFIN-V2 in complex with LAIR1. a Schematic view of A-RIFIN domains (upper, SP: Signal peptide; V1: Variable domain 1; $P^{\star}$ : PEXEL motif; C1: Conserved domain 1; I*: Indel motif; V2: Variable domain 2; TM: Transmembrane domain; C2: Conserved domain 2). Structure of RIFIN (PF3D7_1040300) V2 domain in complex with antibody MGD21 LAIR1 domain is presented as cartoon side view (lower). RIFIN-V2 domain is colored in rainbow. LAIR1 domain is colored in cyan. All secondary structural elements are labeled. $\mathbf{b}$, Surface and pockets on LAIR-1 that accommodate RIFIN-V2 apex. c Detail of RIFIN-LAIR1 interface. Interface residues are shown with side chains as sticks and labeled.

thus reducing the collagen self-reactivity of these antibodies. Overall, these structure-function studies show how B cells expressing antibodies with inserted LAIR1 can reduce collagen affinity without impacting LAIR1 affinity and how native LAIR1 can acquire a $\sim 10$-fold increase in affinity to RIFIN through single amino acid substitutions at either position 151 or 153 ; this affinity increase provides biological context for the affinity matured LAIR1 used in our structural definition of the LAIR1RIFIN interaction as well as insight into the selection and maturation of LAIR1-containing antibodies. Comparing with the affinity matured LAIR1 on the antibodies, the native LAIR1 receptor had a much-reduced affinity to the RIFIN tested in this study, in a similar range to the recently reported RIFIN-LILRB1 interaction ${ }^{7}$. This possibly reflects an optimal balance of parasitic functions between immune receptor activation and avoidance of immune cell attachment.

Delineation of LAIR1-binding sequence signature in RIFIN. Sequence conservation analysis of 158 PF3D7 A-type RIFIN sequences indicated that, although the C254-C265 disulfide bond in the V2 domain is conserved, residues corresponding to the LAIR1-binding region were highly variable (Supplementary Fig. 5c). Phylogenetic analysis of the RIFIN-V2 apex sequence revealed two additional 3D7 RIFINs, referred to as RIF3 (PF3D7_0401300) and RIF4 (PF3D7_0401200), to be in the same branch as the initially identified LA IR1-positive RIFINs (RIF1 and RIF2) (Fig. 3a). A similar approach also identified seven RIFINs in non-3D7 strains. Co-immunoprecipitation (co-IP) assays confirmed LAIR1 interaction with the newly identified 3D7 RIFINs, RIF3 and RIF4 (Fig. 3b), and with three of the non-3D7 RIFINs (Supplementary Fig. 6a, b).

To provide insight into the recognition of these RIFINs, we determined the structure of the RIF3-V2 domain in complex with
MGD21 LAIR1 at $2.5 \AA$ resolution (Table 1). The RIF3-LAIR1 structure resembled that of RIF1-LAIR1 with an overall rmsd of $1.46 \AA$ over $546 \mathrm{Ca}$ atoms (Fig. 3c), despite clear diversity in the primary RIFIN sequences at the binding interface (Fig. 3d). Structural analysis and sequence comparison of the LAIR1-RIFIN complex suggested a set of four conserved residues contributing to LAIR1-binding capacity, including residues D247, C254, C265 and R268. D247 forms hydrogen bonds with Q284 to stabilize interaction between $\alpha 4$ and $a 6$; C254-C265 forms a disulfide bond connecting $\alpha 4$ and $\alpha 5$; and R268 not only connects $\alpha 5-\alpha 6$ by hydrogen-bonding with Q284, but also forms multiple interactions with LAIR1 by $\pi$-stacking with Y152 $2_{\text {LAIR } 1}$ and by hydrogen bonding with the main chain carbonyl of Y150 ${ }_{\text {LAIR1 }}$ (Figs. $3 \mathrm{c}$ and $2 \mathrm{~d})$. We further verified the contribution of the four conserved RIFIN residues to the LAIR1-binding capacity by co-IP assay with alanine mutations. The D247A mutation significantly reduced affinity between RIFIN and LAIR1, and all other single mutations completely knocked out the LAIR1 binding capacity of RIFIN (Fig. 3e).

To facilitate the identification of additional LAIR1-binding RIFIN, we incorporated the above four conserved residues in a LAIR1 recognition signature (Fig. 4a), and further identified 47 signature-matched RIFINs from the Plasmodium genome database (Fig. 4b). By comparison, a "three-residue" signature without D247 identified 92 signature-matched RIFINs (Supplementary Data 1). Co-immunoprecipitation confirmed LAIR1 binding for 20 of the 47 RIFINs with the four-residue signature (Fig. $4 \mathrm{c}$ and Supplementary Fig. 7a). A fluorescence-activated cell sorting (FACS) assay further showed that the surface of Expi293F cells expressing full-length constructs of four selected RIFIN whose binding to LAIR1 was confirmed by immunoprecipitation to be stained by both MGD21 antibody and native LAIR1, but not by LILRB1-inserted antibodies, MDA1, MDB1, and MDC $1^{12}$ or by a negative control antibody (Fig. 4d and Supplementary Fig. 7b). 
a Superimposition of LAIR1 in free and RIFIN-bound structures

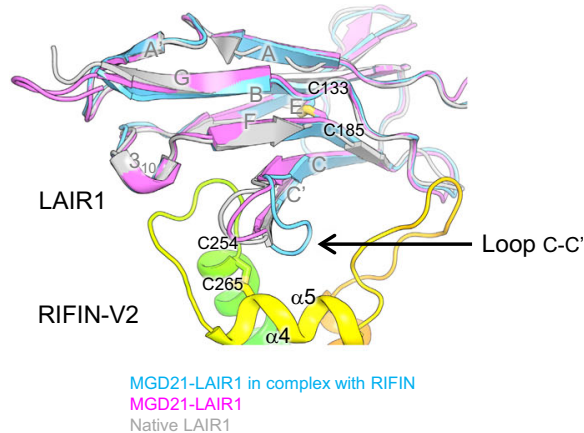

b Alignment of LAIR1 sequences

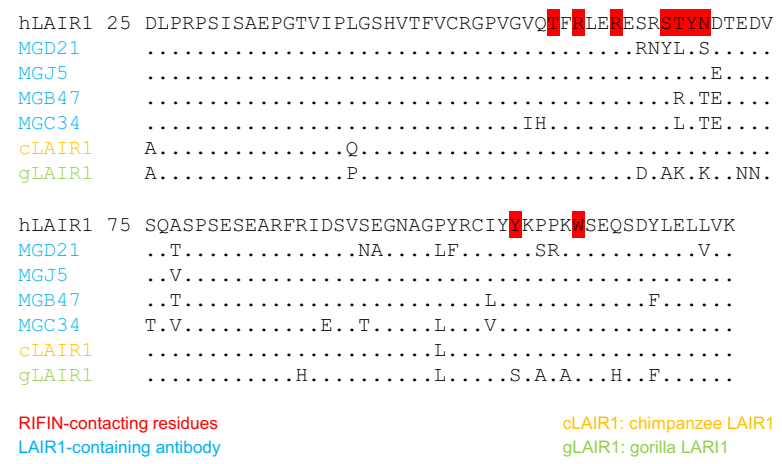

C Mutations contributing to the LAIR1 affinity maturation to RIFIN
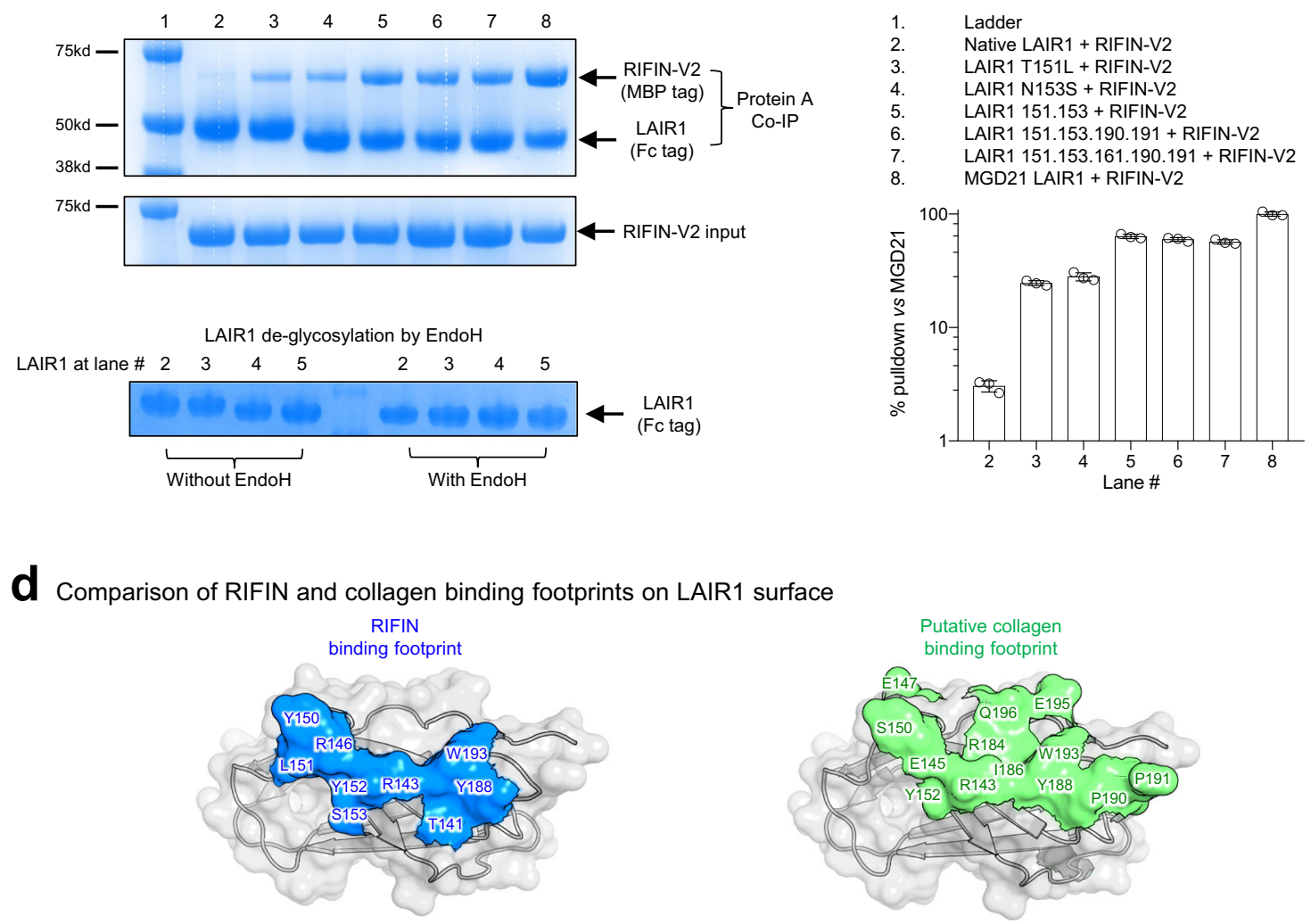

Fig. 2 Structural and sequence analysis of various LAIR1 constructs identifies RIFIN-binding essential. a Conformational change on LAIR1 c-c' loop to enable RIFIN binding. b Alignment of LAIR1 sequences. (MGD21, hLAIR1: native human LAIR1, MGJ5, MGB47, MGC34, gLAIR1: gorilla LAIR1). c Accumulation of critical mutations allows affinity maturation of LAIR1 to RIFIN (upper left panel: SDS-PAGE image; lower left: LAIR1 de-glycosylation by EndoH; upper right panel: SDS-PAGE sample loading list; lower right panel: Quantification of left panel by bar graph. Data are presented as mean values \pm SD). $n=3$ independent measurements. The SDS-PAGE runs were repeated at least twice. d RIFIN binding footprint overlapped that of collagen on LAIR1 surface. Note: LAIR1 residue numbering is based on MGD21 construct, which has 84 shift to that of native LAIR1.

Taxonomy analysis indicated the 27 RIFINs with confirmed LAIR1 binding to be from multiple P. falciparum field strains or non-human primate-specific Plasmodium species, including P. sp. gorilla clade G1, which infects gorillas, and Plasmodium reichenowi, which infects chimpanzees (Fig. 4e).

\section{Discussion}

Only a small subset of RIFIN family members can interact with LAIR1 ${ }^{6}$; however, the structural and bioinformatic analyses performed here allowed us to delineate a sequence signature for LAIR1-interacting RIFINs, by delineating critical residues at the RIFIN apex and their contribution to LAIR1 specificity. Binding to LAIR1 was confirmed in 20 of the 47 signature-matched RIFINs. These results demonstrate LAIR1-binding RIFINs to exist in multiple, evolutionarily-related, Plasmodium species capable of infecting humans, chimpanzees and gorillas, suggesting a link in the evolutionary origin of the human malaria parasite ${ }^{13}$.

The "razor and cartridge" mode of interaction between RIFIN and LAIR1 that we define here may constitute a general structural mechanism used by RIFIN to target inhibitory receptors ${ }^{12}$. The interface between RIFIN-V2 apex and LAIR1 C-C' region largely comprises hydrophobic interactions and backbone-mediated hydrogen bonds, and the interface is facilitated by an induced conformational fit of flexible RIFIN-V2 apex loops. These 
a Phylogenic tree of 3D7 RIFIN-V2 apex

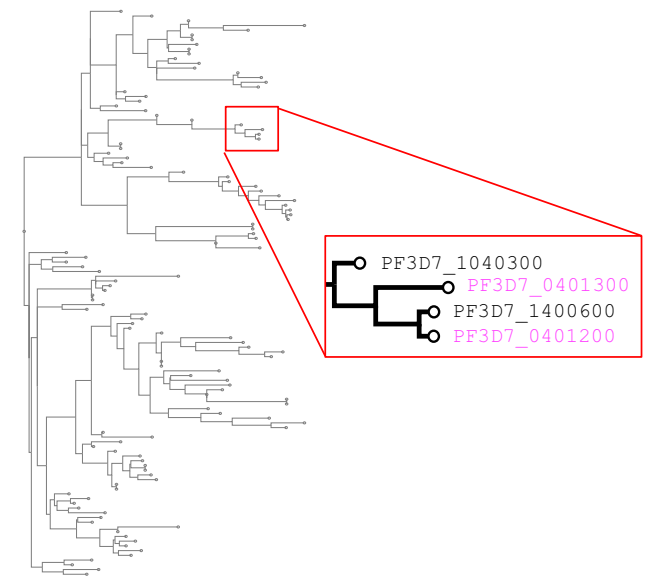

C Superimposition of two RIFIN-LAIR1 complexes

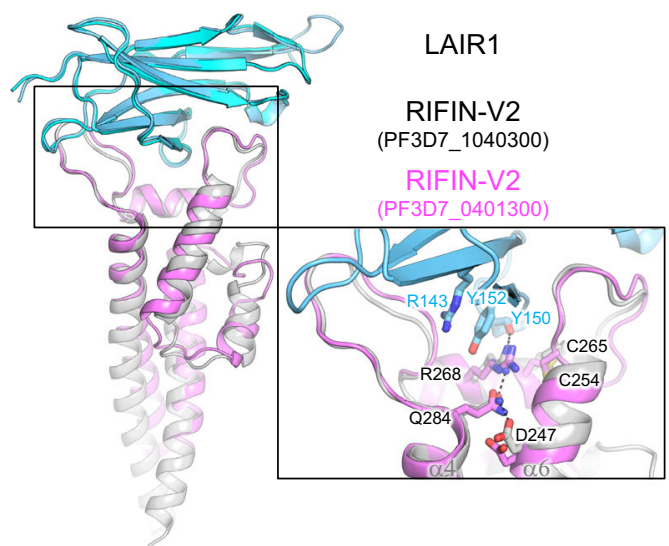

b Binding of two new PF3D7 RIFINs to LAIR1 ${ }_{\text {MGD21 }}$

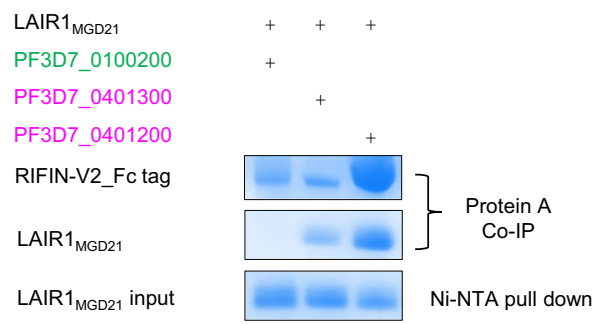

d RIFIN-V2 domain sequence alignment

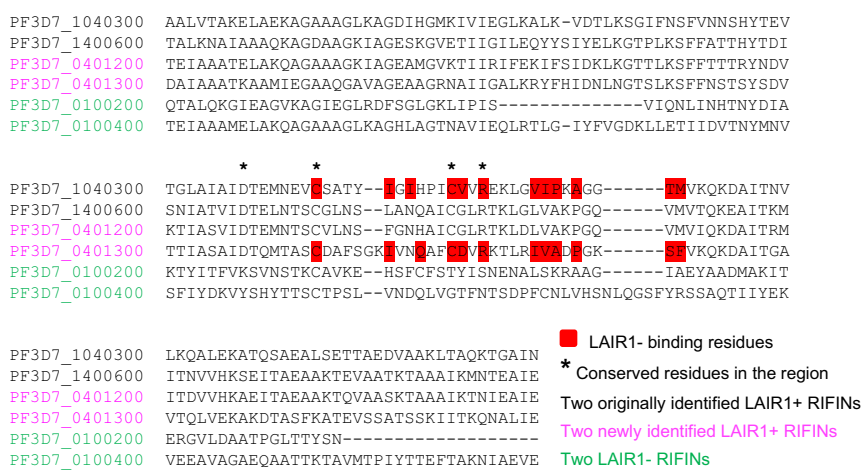

C Co-IP with mutants in RIFIN-V2 conserved residues

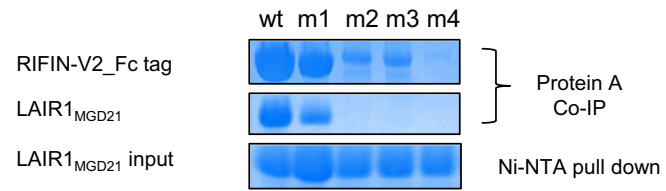

Fig. 3 LAIR1 targets a conserved interface within a subset of RIFIN family members. a Phylogenic analysis of plasmodium strain 3D7 RIFIN-v2 apex identified two additional RIFINs in the same branch as known LAIR1-binding RIFINs. b Co-IP assay confirmed the MGD21.LAIR1-binding by the two new RIFINs. The SDS-PAGE runs were repeated at least twice. c Superimposition of two RIFIN-LAIR1 complex structures. d Sequence comparison of MGD21positive (cyan and magenta) and MGD21-negative (green) RIFINs V2 region, with LAIR1-binding region highlighted in red and conserved residues near the binding region marked with asterisks $\left(^{\star}\right)$. e Co-IP with Alanine mutants in RIFIN-V2 four conserved residues. wt: wildtype PF3D7_1040300, m1: D247A, m2: C254A, m3: C265A, m4: R268A. The SDS-PAGE runs were repeated at least twice.

structural and chemical properties appear to allow for promiscuous interactions between the LAIR1 receptor and diverse Plasmodium RIFINs, suggesting RIFIN-mediated LAIR1-targeting may be a conserved strategy allowing for immune suppression across multiple Plasmodium species of malignant malaria, possibly inherited from common ancestors.

In terms of immune recognition of LAIR1-targeting RIFINs, we note that the antigenic diversity of RIFINs $s^{3,4,14}$, the limited surface coincidence of different rifin genes ${ }^{5}$, and the rapid switching of variant surface antigens ${ }^{15}$ may allow for effective evasion of host immune targeting. Collectively, the presence of multiple LAIR1-binding RIFINs and their polymorphism provides evidence for the importance of targeting inhibitory receptors as well as a means to escape immune responses targeting specific RIFINs. The extensive sequence diversity of the LAIR1binding surface of RIFIN-even among those RIFINS that we demonstrated to be positive for LAIR1 binding by co-immunoprecipitation-along with the sequence conservation of LAIR1s from hosts infected by malaria (Fig. 2b) highlights the complex polymorphic interactions that malaria parasites make with host factors to enhance their survival. In this context, we note that receptor-based antibodies, such as the LAIR1-inserted antibodies analyzed here, have been optimized by B cell-based mechanisms of affinity maturation (Fig. 2c) and may thus exemplify "lessescapable" solutions for pathogen recognition.

\section{Methods}

Construct and protein preparation. LAIR1 (wild type and mutants), antibodies (MGC34, MGD21, MGJ5, MGM1) and RIFIN genes were synthesized and subcloned (GenScript, NJ) into pVRC8400 vectors, with HRV3C cleavable His or Fc tag. DNAs were transfected into either Expi293F cells or Expi293 GnTI- cells (Thermo Fisher) using Turbo293 transfection reagent (Speed BioSystems). Six days post transfection, culture supernatants were harvested, and affinity purified with cOmplete $^{\text {Tx }}$ His-Tag Purification Resin (Roche) or protein A resin (GE) by following manufacture's protocols.

Affinity measurement. Affinity of native LAIR1 and MGD21 to RIFIN_V2 domain was assessed using a fortéBio Octet Red384 instrument. His-tagged RIFIN V2 domain was immobilized on Ni-NTA biosensors, then dipped into either LAIR1 or MGD21 in the twofold concentration series. Sensorgrams of the concentration series were corrected with corresponding blank curves and fitted globally with Octet evaluation software using a 1:1 Langmuir model of binding.

Co-IP assay. Protein complex co-immunoprecipitation were performed by incubating condition media with protein A resin for one hour at room temperature. Fctagged RIFIN or LAIR1 were pulled down by protein A resin, while the non Fctagged proteins were co-pulled down. The co-IP protein complexes were analyzed with SDS-PAGE and visualized by staining with Coomassie blue. SDS-PAGE gel band intensity was quantified with ImageJ (https://imagej.nih.gov/). 
Table 1. Statistics of $\mathrm{X}$-ray diffraction data collection and structural refinement.

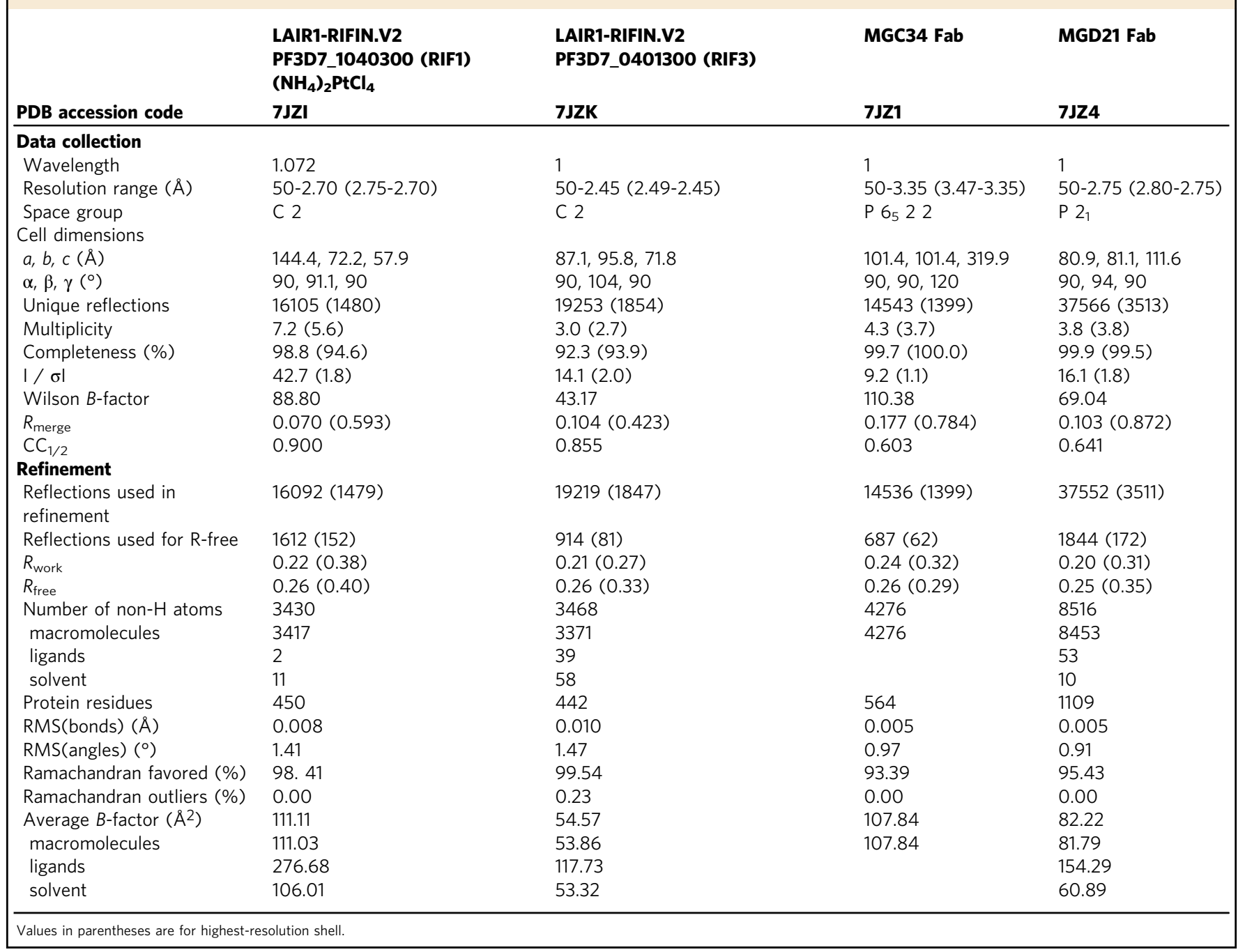

Negative-staining EM analysis. Samples were diluted with a buffer containing 20 mM HEPES, pH 7.0, $150 \mathrm{mM} \mathrm{NaCl}$, adsorbed to a freshly glow-discharged carbonfilm grid, washed with the above buffer, and stained with $0.7 \%$ uranyl formate. Images were collected at a magnification of 100,000 using SerialEM ${ }^{16}$ on a FEI Tecnai T20 microscope equipped with a $2 \mathrm{k} \mathrm{x} 2 \mathrm{k}$ Eagle CCD camera and operated at $200 \mathrm{kV}$, or at a magnification of 57,000 using EPU on a ThermoFisher Talos F200C microscope equipped with a $4 \mathrm{k}$ x $4 \mathrm{k}$ CETA $16 \mathrm{M}$ camera and operated at $200 \mathrm{kV}$. The pixel size was $2.2 \AA$ for the Eagle CCD camera and $2.5 \AA$ for the CETA camera. Particles were picked using e2boxer from the EMAN $2^{17}$ software package. Reference-free $2 \mathrm{D}$ classification was performed using EMAN2 and SPIDER ${ }^{18}$. 3D reconstruction was performed using SPIDER, cryoSPARC ${ }^{19}$ and FREALIGN ${ }^{20}$, with initial 3D references generated with EMAN2.

Crystal screening and X-ray crystallographic data collection. To facilitate crystallization, proteins and complexes were treated with HRV3C to remove affinity tags and further purified by SEC with Superdex 200 chromatography column (GE) in the HEPES buffer ( $5 \mathrm{mM}$ HEPES pH7.5 and $150 \mathrm{mM} \mathrm{NaCl}$ ). Crystallization conditions were screened using Hampton Research, Wizard, and QIAGEN crystal screening kits. Crystal plates were set up using a Mosquito crystallization robot. Crystals initially observed from the wells were manually reproduced. MGC34 crystal grew in $0.05 \mathrm{M} \mathrm{CaCl}_{2}, 0.1 \mathrm{M}$ BIS-TRIS pH 6.5 and 30\% v/v PEG MME 550. MGD21 crystal grew in $0.1 \mathrm{M} \mathrm{MgCl}_{2}, 0.1 \mathrm{M}$ Tris $\mathrm{HCl} \mathrm{pH} 8.5,20 \%$ PEG 3350 and $25 \%$ PEG 400. RIF1-LAIR1 complex crystal grew in $0.085 \mathrm{M}$ HEPES pH 7.5, 17\% PEG 4000, 15\% Glycerol and 8.5\% Isopropanol. RIF3-LAIR1 complex crystal grew in $0.1 \mathrm{M} \mathrm{NaCl}, 0.1 \mathrm{M}$ Tris $\mathrm{HCl}$ pH 8.5 and 20\% PEG 3350. Optimized crystals were cryoprotected in $30 \%$ glycerol and flash-frozen in liquid nitrogen. Platinum derivatives of RIF1-LAIR1 complex crystal were prepared by soaking the crystal in well solution supplemented with various concentration of $\left(\mathrm{NH}_{4}\right)_{2} \mathrm{PtCl}_{4}$ for $1 \mathrm{~h}$. Data were collected at a wavelength of $1.00 \AA$, except Platinum derivative (1.072 $\AA$ ), at the SER-CAT beamline ID-22 (Advanced Photon Source, Argonne National Laboratory).
Structural determination. Diffraction data were processed with the HKL2000 suite ${ }^{21}$. Phasing solution of RIF1-LAIR1 structure were determined by MR-SAD in Phenix ${ }^{22}$ with combination of molecular replacement (LAIR1 template: 3KGR) and Platinum signal. Other structural solution was obtained by molecular replacement with Phaser in Phenix using search models consisting of the variable region generated with PIGSpro ${ }^{23}$ and the constant regions. Model building was carried out with $\mathrm{Coot}^{24}$. Refinement was carried out with Phenix. Data collection and refinement statistics are shown in Table 1.

Bioinformatic analysis. Pairwise RIFIN V2 structural comparison was perform with FATCAT server ${ }^{25}$. RIFIN structural conservation against structures in the Protein Data Bank was analyzed with Dali server ${ }^{26}$. To calculate sequence conservation, all type A RIFIN sequences of strain 3D7 were downloaded from NCBI. Duplicate sequences were removed. The remaining 117 sequences were aligned with clustalW ${ }^{27}$. The Shannon entropy was calculated for each residue, yielding a minimum value of 0.282 and a maximum value of 2.352 . Shannon entropy values were then assigned to the B-factor column of the PDB file and the structure was colored by Shannon entropy using PyMOL. The signature of LAIR1 binding RIFIN was defined by the conserved amino acids that contribute to the interface and the structural integrity of V2 apex. The complete protein sequences of Malaria RIFIN were downloaded at Nov-06-2019 from UniProt, from which the V2 domain sequences were extracted for signature analysis. Phylogenetic analysis was first performed by aligning the V2 domain sequences with $\mathrm{Mafft}^{28}$, then a neighbor joining tree was constructed by using clustalW ${ }^{27}$. Python library ETE $^{29}$ was used to visualize phylogenetic tree.

FACS analysis. To validate whether selected RIFINs are the antigens for LAIR1containing antibodies MGD21 or germ-line reverted MGD21-UCA, gene encoding V2 region of RIFIN candidates were produced by gene synthesis (Genescript) and cloned into pDisplay vector (Invitrogen) that contains a hemagglutinin (HA) tag. 
a RIFIN signature for LAIR1 recognition

$$
\text { D. }\{6\} C \cdot\{10,12\} C \cdot\{2\} R
$$

\section{b}

Phylogenic tree analysis of signature-matched RIFIN-V2

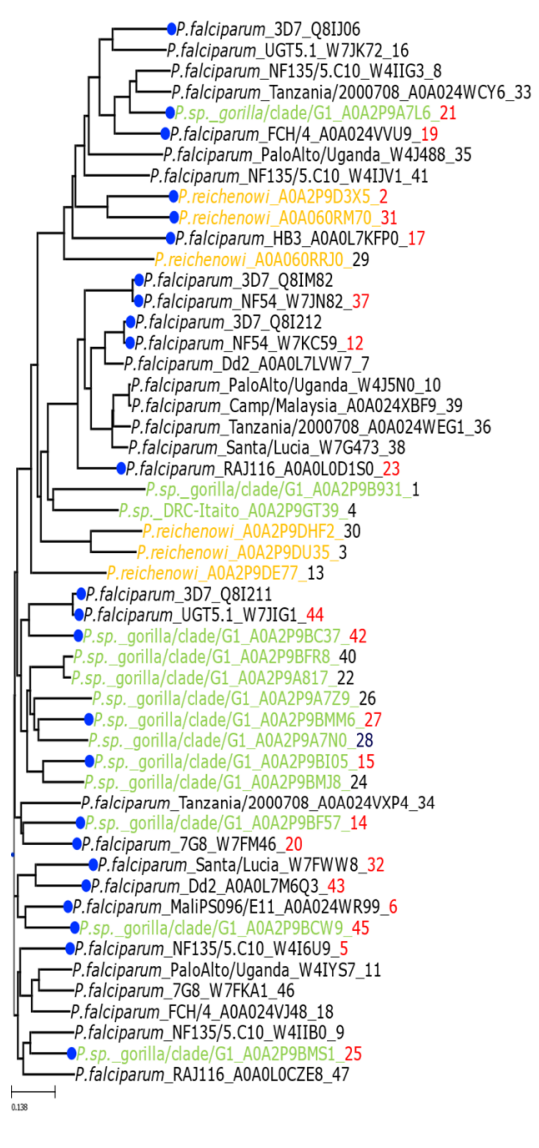

- LAIR1 positive RIFINs

$P$. falciparum

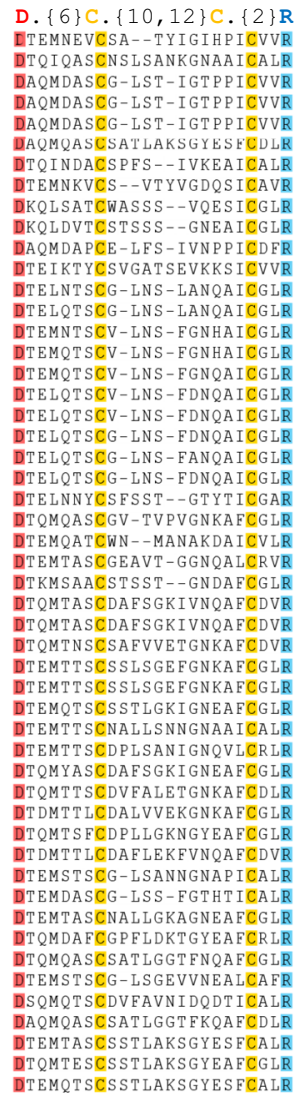

DTEMQTSCSSTLAKSGYESFCALR

P.sp.gorilla clade G1
C LAIR1 ${ }_{\text {MGD21 }}$ co-IP by Fc-tagged RIFIN-V2
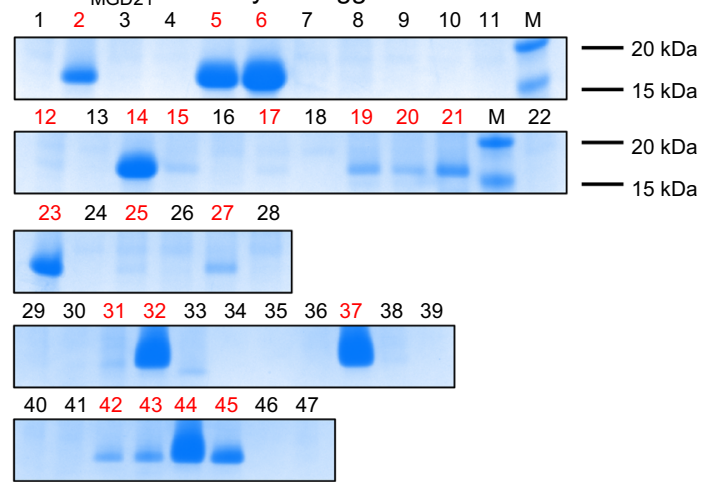

d

FACS analysis of RIFIN-LAIR1/LILRB1 interaction
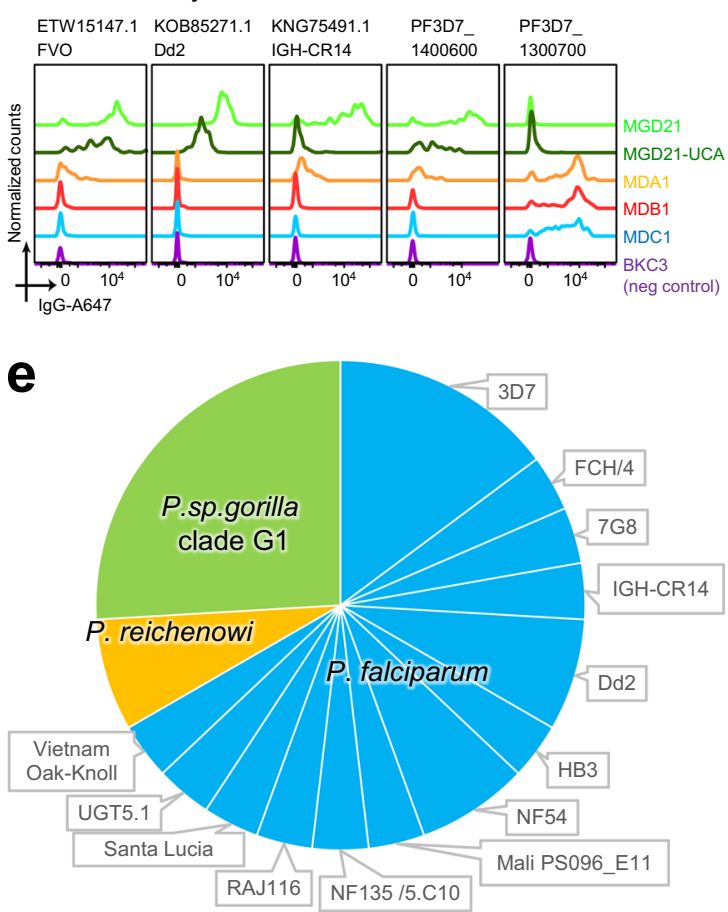

Fig. 4 Signature-based search identifies LAIR1-binding RIFINs from Plasmodium sequences. a Structure-based delineation of LAIR1 binding signature on RIFIN. b Phylogenic tree of V2 domain showing name and apex sequences of signature-matched RIFINs. Blue circles indicate the empirically confirmed LAIR1-positive RIFIN. Ape-infecting strains are highlighted in yellow or green, and $P$. falciparum strains in black. The number at the end of each name indicates its sequence in (c). c Coomassie blue-stained SDS-PAGE showing LAIR1 MGD21 co-immunoprecipitation (Co-IP) by Fc-tagged RIFIN-V2 constructs identified by the signature. Confirmed LAIR1-binding RIFIN ID are highlighted in red. Lanes marked with "M" are molecular ladders showing 15-20 kDa bands. The SDS-PAGE runs were repeated at least twice. d FACS analysis example of RIFIN-LAIR1 (MGD21 and MGD21-UCA) or RIFIN-LILRB1(MDA1, MDB1, and MDC1) interaction. e Pie chart summary of 27 experimentally confirmed LAIR1-positive RIFINs, colored by three Plasmodium species ( $P$. falciparum and two ape-specific species).

RIFIN-containing pDisplay vectors were transiently transfected into Expi293F cells respectively (ThermoFisher Scientific) using PEI. $72 \mathrm{~h}$ post-transfection, cells expressing RIFINs were collected and stained with MGD21, MGD21UCA, LILRB1containing antibodies MDA1, B1, C1 ${ }^{12}$ or control IgG antibodies BCK3 and tested by flow cytometry. Briefly, $5 \mu \mathrm{g} / \mathrm{ml}$ of testing antibodies were added to the RIFINtransfected cells for $30 \mathrm{~min}$ at $4{ }^{\circ} \mathrm{C}$ and followed by washing steps before staining with secondary of $2.5 \mu \mathrm{g} / \mathrm{ml}$ of Alexa Fluor 647-conjugated goat anti-human IgG antibody (Jackson ImmunoResearch, catalog no. 109-606-170). The cells were washed again, and then stained with $5 \mu \mathrm{g} / \mathrm{ml}$ of rabbit anti-HA tag for the same condition followed by second washing before adding Alexa Fluor 488-conjugated goat anti-rabbit IgG (Life Technologies, catalog no. A11034) for the detection of RIFIN binding. Dead cells were excluded by gating. FACS was performed in BD FACSCanto I (Cat no. 337175). Gating followed the standard gating procedures. Under the pseudocolor plot, live cells were in grouped in clear population in the FSC/SSC plot. The Singlets were gated by eliminating the shades of the edge. RIFIN-HA-488 positive population was determined by comparing to the unstained cells. IgG-A647 was visualized using histogram. FACS Diva (version 6.2) was used for acquisition of samples. Flow-Jo (version 10.1) was used for all the FACS analyses. A figure exemplifying the gating strategy is provided in the Fig S7b.

Reporting summary. Further information on research design is available in the Nature Research Reporting Summary linked to this article.

\section{Data availability}

Coordinates for the crystal structures determined in this study have been deposited in the Protein Data Bank as PDB 7JZ1, 7JZ4, 7JZI and 7JZK. PDB 3KGR and 6ZDX were downloaded from Protein Data Bank. These data were analyzed in Figs. 1, 2, 3 and Supplementary Figs. 1, 5. Raw data (SDS-PAGE gel image) associated with Figs. 2c, 3b, 3e, $4 c$, S6 and S7a are provided in Source data file. Plasmodium genome database can be accessed on the web (plasmodb.org). All relevant data are provided with this paper and also available from the authors. Source data are provided with this paper. 
Received: 8 February 2021; Accepted: 11 June 2021;

Published online: 09 July 2021

\section{References}

1. World Health Organization. World malaria report 2019, (2019).

2. Kyes, S., Horrocks, P. \& Newbold, C. Antigenic variation at the infected red cell surface in malaria. Annu Rev. Microbiol. 55, 673-707 (2001).

3. Fernandez, V., Hommel, M., Chen, Q., Hagblom, P., \& Wahlgren, M.Small, clonally variant antigens expressed on the surface of the Plasmodium falciparum-infected erythrocyte are encoded by the rif gene family and are the target of human immune responses. J. Exp. Med 190, 1393-1404 (1999).

4. Kyes, S. A., Rowe, J. A., Kriek, N. \& Newbold, C. I. Rifins: a second family of clonally variant proteins expressed on the surface of red cells infected with Plasmodium falciparum. Proc. Natl Acad. Sci. USA 96, 9333-9338 (1999)

5. Goel, S. et al. RIFINs are adhesins implicated in severe Plasmodium falciparum malaria. Nat. Med 21, 314-317 (2015).

6. Saito, F. et al. Immune evasion of Plasmodium falciparum by RIFIN via inhibitory receptors. Nature 552, 101-105 (2017).

7. Harrison T. E., et al. Structural basis for RIFIN-mediated activation of LILRB1 in malaria. Nature 587, 309-312 (2020).

8. Tan, J. et al. A LAIR1 insertion generates broadly reactive antibodies against malaria variant antigens. Nature 529, 105-109 (2016).

9. Pieper, K. et al. Public antibodies to malaria antigens generated by two LAIR1 insertion modalities. Nature 548, 597-601 (2017).

10. Robbiani, D. F. \& Nussenzweig, M. C. A new way to diversify antibodies by DNA transposition. Cell 164, 601-602 (2016).

11. Brondijk, T. H. et al. Crystal structure and collagen-binding site of immune inhibitory receptor LAIR-1: unexpected implications for collagen binding by platelet receptor GPVI. Blood 115, 1364-1373 (2010).

12. Chen, Y. et al. Structural basis of malaria RIFIN binding by LILRB1containing antibodies. Nature 592, 639-643 (2021).

13. Liu, W. et al. Origin of the human malaria parasite Plasmodium falciparum in gorillas. Nature 467, 420-425 (2010).

14. Bultrini, E. et al. Revisiting the Plasmodium falciparum RIFIN family: from comparative genomics to 3D-model prediction. BMC Genomics 10, 445 (2009).

15. Guizetti, J. \& Scherf, A. Silence, activate, poise and switch! Mechanisms of antigenic variation in Plasmodium falciparum. Cell Microbiol. 15, 718-726 (2013).

16. Mastronarde, D. N. Automated electron microscope tomography using robust prediction of specimen movements. J. Struct. Biol. 152, 36-51 (2005).

17. Tang, G. et al. EMAN2: an extensible image processing suite for electron microscopy. J. Struct. Biol. 157, 38-46 (2007).

18. Frank, J. et al. SPIDER and WEB: processing and visualization of images in 3D electron microscopy and related fields. J. Struct. Biol. 116, 190-199 (1996).

19. Punjani, A., Rubinstein, J. L., Fleet, D. J. \& Brubaker, M. A. cryoSPARC: algorithms for rapid unsupervised cryo-EM structure determination. Nat. Methods 14, 290-296 (2017).

20. Grigorieff, N. FREALIGN: high-resolution refinement of single particle structures. J. Struct. Biol. 157, 117-125 (2007).

21. Otwinowski, Z. \& Minor, W. Processing of X-ray diffraction data collected in oscillation mode. Methods Enzymol. 276, 307-326 (1997).

22. Adams, P. D. et al. PHENIX: a comprehensive python-based system for macromolecular structure solution. Acta Crystallogr D. Biol. Crystallogr 66, 213-221 (2010).

23. Marcatili, P., Rosi, A. \& Tramontano, A. PIGS: automatic prediction of antibody structures. Bioinformatics 24, 1953-1954 (2008).

24. Emsley, P. \& Cowtan, K. Coot: model-building tools for molecular graphics. Acta Crystallogr D. Biol. Crystallogr 60, 2126-2132 (2004).

25. Li, Z., Jaroszewski, L., Iyer, M., Sedova, M. \& Godzik, A. FATCAT 2.0: towards a better understanding of the structural diversity of proteins. Nucleic Acids Res. 48, W60-W64 (2020).

26. Holm, L. \& Rosenstrom, P. Dali server: conservation mapping in 3D. Nucleic Acids Res. 38, W545-W549 (2010).
27. Thompson, J. D., Higgins, D. G. \& Gibson, T. J. CLUSTAL W: improving the sensitivity of progressive multiple sequence alignment through sequence weighting, position-specific gap penalties and weight matrix choice. Nucleic Acids Res. 22, 4673-4680 (1994).

28. Katoh, K. \& Standley, D. M. MAFFT multiple sequence alignment software version 7: improvements in performance and usability. Mol. Biol. Evol. 30, 772-780 (2013)

29. Huerta-Cepas, J., Serra, F. \& Bork, P. ETE 3: reconstruction, analysis, and visualization of phylogenomic data. Mol. Biol. Evol. 33, 1635-1638 (2016).

\section{Acknowledgements}

We thank J. Stuckey for assistance with figures and members of the Virology Laboratory, Vaccine Research Center, for discussion and comments. This work was supported by the Intramural Research Program of the Vaccine Research Center, National Institution of Allergy and Infectious Diseases, NIH, by the GenScript Innovation grant GS-IG-2018-003 (KX), and by federal funds from the Frederick National Laboratory for Cancer Research, NIH, under Contract HHSN261200800001 (TS, YT). Use of sector 22 (Southeast Region Collaborative Access team) at the Advanced Photon Source was supported by the US Department of Energy, Basic Energy Sciences, Office of Science (contract W-31-109-Eng-38).

\section{Author contributions}

K.X. conceived the project. K.X., A.L., and P.D.K. supervised the research and wrote the manuscript. K.X., Y.W. and K.L. determined crystal structures. Y.T., K.X., and T.S. performed EM analysis. C.-H.S. and K.X. defined sequence signatures, K.F. analyzed RIFIN sequence conservation under the supervision of G.-Y.C., Y.C. performed FACS analysis. J.G., T.Z., and L.P. designed protein constructs. B.Z., R.V., and Y.Y. assisted with cell culture. S.W. assisted with manuscript editing.

\section{Funding}

Open Access funding provided by the National Institutes of Health (NIH).

\section{Competing interests}

Y.C., A.L., and L.P. are currently employees of Vir Biotechnology Inc. and may hold shares in Vir Biotechnology Inc. The other authors declare no competing interests.

\section{Additional information}

Supplementary information The online version contains supplementary material available at https://doi.org/10.1038/s41467-021-24291-6.

Correspondence and requests for materials should be addressed to K.X. or P.D.K

Peer review information Nature Communications thanks the anonymous reviewers for their contribution to the peer review of this work.

Reprints and permission information is available at http://www.nature.com/reprints

Publisher's note Springer Nature remains neutral with regard to jurisdictional claims in published maps and institutional affiliations.

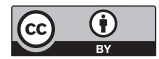

Open Access This article is licensed under a Creative Commons Attribution 4.0 International License, which permits use, sharing, adaptation, distribution and reproduction in any medium or format, as long as you give appropriate credit to the original author(s) and the source, provide a link to the Creative Commons license, and indicate if changes were made. The images or other third party material in this article are included in the article's Creative Commons license, unless indicated otherwise in a credit line to the material. If material is not included in the article's Creative Commons license and your intended use is not permitted by statutory regulation or exceeds the permitted use, you will need to obtain permission directly from the copyright holder. To view a copy of this license, visit http://creativecommons.org/ licenses/by/4.0/.

This is a U.S. government work and not under copyright protection in the U.S.; foreign copyright protection may apply 2021 